\title{
PATHOGENETIC ASPECTS OF MYELODISPLASTIC SYNDROMES
}

\author{
F. Lauria (lecturer), A. Gozzetti, D. Raspadori, A. Petrini, M. Defina
}

\author{
Ematology and Transplant Unit, Dept. of Clinical Medicine and Immunological Sciences \\ University of Siena
}

\begin{abstract}
The patogenesis of MDS is complex and remain elusive. Figure 3 shows a hypothetical model. The proposed models agree that a multistep process occurs through which a hematopoietic stem cell is mutated and attains a growth advantage. This may occur as a result of environmental damage or inherited predisposition. The mutated clone is associated with morphological dysplasia, impaired differentiation and genomic instability. Cytokine secretion and apoptotic pathways are altered and as well as may be impairment of immune responses. Presumably, in the early stages, increased production of proapoptotic cytokines leads to excessive apoptosis, correlating clinically with cytopenias and a cellular bone marrow. As the disease progresses, further genetic and epigenetic events occur, resulting in decreased apoptosis, clonal expansion and progression to AML. Clinical testing of a number of molecules that affect these myriad molecular mechanisms is currently being done, characterization of genomic expression patterns will inform both diagnosis and prognostication. Further insight into the molecular mechanisms of MDS will provide an avenue for more tailored and effective therapy in the future.
\end{abstract}

\section{INTRODUCTION}

The myelodysplastic syndromes (MDS) comprise a heterogeneous group of clonal hematopoietic stem cell disorders characterized by ineffective hematopoiesis and a variable risk of transformation to acute myeloid leukemia (AML) (1).

It has been suggested that MDS arise from a hematopoietic stem cell that has suffered irreversible DNA damage. In a early phase of disease, impaired differentiation and increased apoptosis predominate, but as disease progression occurs, increased proliferation and accumulation of immature cells result.

Diagnosis and current classification systems (e.g. FAB or WHO) are based on findings in peripheral blood and expecially in the bone marrow as well as on cytogenetic abnormalities $(2,3)$. In particular, diagnosis of MDS should be suspected in any patient with unexplained cytopenia(s) or monocytosis. A careful examination of peripheral blood as well as bone marrow smears show evident morphological abnormalities in one or more lineages. Fig. 1 and 2 depict some of the morphological patterns frequently seen in these diseases. This chapter will summarize some of the observations on the numerous biologic mechanisms implicated in the pathophysiology of MDS, including intrinsic progenitor abnormalities, epigenetic changes, abnormal apoptosis machinery, immunologic influences, abnormal signal transduction pathways and the role of the bone marrow microenvironment.

\section{GENOMIC INSTABILITY}

MDS is thought to derive from the somatic mutation of hematopoietic progenitor cell. Confirmation of the clonality in these patients has been demonstrated by analysis of cytogenetic and $\mathrm{X}$-chromosome inactivation studies $(4,5)$. However, scientific evidence has grown in support of the concept that the cytogenetic abnormalities, seen so frequently in MDS, may be acquired during disease progression rather than reflecting the initial clonal event $(5,6)$. Whether a primary or secondary event, genomic instability, as evidenced by karyotypic changes common in MDS, is thought to play an important role in disease pathogenesis.

Cytogenetic abnormalities in MDS result from the accumulation of genomic damage, failure to repair such damage, or both. Although the etiology of most cases of MDS is unknown, exposure to genotoxic agents such as benzene, radiation, or prior treatment with chemotherapeutic agents is known to increase the risk of developing MDS (7). Other environmental agents that may increase the risk include smoking, heavy metals, pesticides, fertilizers, petroleum products, and organic chemicals $(8,9,10)$.

Another possible mechanism underlying genomic instability involves telomere dynamics, and the enzyme telomerase. Telomere erosion may result in chromosome end fusion and subsequent chromosome instability. Shortened telomere length has been reported to be associated with poor prognosis in patients with MDS $(11,12)$.

\section{EPIGENETIC Modifications}

While genetic alterations are critical in the pathogenesis of MDS, epigenetic changes also contribute significantly to the disease phenotype. A modern definition of epigenetics refers to " modifications in gene expression that are brought about by heritable, but potentially reversible changes in chromatin structure and/or DNA methylation" (13). Epigenetic changes include methylation of cytosine residues followed by a guanine base (DNA methylation) and post-translational modifications of histones that lead to alteration in chromatin structure at specific gene loci, which in turn determine the transcriptional output of gene.

Methylation of $\mathrm{CpG}$ dinucleotides concentrated in the promoter regions of some genes (so-called ' $\mathrm{CpG}$ island') results in the functional inactivation of those genes without alteration in the primary sequence. Both hypomethylation and hypermethylation of genome have been observed in hematological malignancies. Hypomethylation of Multi Drug Resistance I (MDR I) gene has been described in AML samples and was shown to correlate with increased expression as measured by reverse transcripion polymerase chain reaction (RT-PCR), possibly contributing to multidrug resistance in these patients $(14,15)$. Hypomethylation of cmyc and myeloperoxidase have also been described in sample from patients with primary AML and AML arising from MDS (16).

While mutations in cell cycle control genes such as p15, 
p16, and p19 have rarely been described in MDS, hypermethylation of p 15 is common. Hypermethylation of the pl5INK4B gene promoter has also been observed in $30-50 \%$ of MDS cases and a correlation with the percentage of bone marrow blasts has been shown $(17,18)$. p15INK4B is a cyclin-dependent kinase inhibitor that is critical in regulating the Gl phase of the cell cycle. Its activation is downstream of the TGF$\triangle / S M A D$ pathway. This suggests that one mechanism of proliferation of leukemic cells is escape of regulation of Gl phase of cell cycle. Further evidence for the importance of this event in MDS pathogenesis derives from the observation that the degree of methylation correlates with the risk of evolution to AML and clinical prognosis (17). Other genes frequently methylated and silenced in myeloid malignancies include E-Cadeherin, RAR $\beta$, and SOCS-1 $(19,20)$.

\section{Abnormalities in Signaling Pathway}

\section{Growth FACTORS}

The ineffective hematopoiesis that is characteristic of MDS has led to the investigation of pathways involved in transducing signals from erythropoietin (EPO) and other growth factors. When bone marrow cells from MDS patients are cultured in colony forming assay in the presence of EPO, erythroid colony formation is reduced in comparison to normal controls $(21,22)$. EPO signaling involves a complex cascade of events beginning with the binding of EPO to the erythropoietin receptor (EPO -R). Upon binding EPO, the Janus Kinase, JAK2, is activated $(23,24)$. JAK2 activation leads to downstream tyrosine phosphorylation of a number of proteins. Activation of signal transducer and activator of transcription 5 (STAT5), a downstream protein of JAK2 that is thought to be important in EPO signaling, is impaired in MDS (25). This observation, combined with the other reports of normal presence of EPO-R in MDS patients, indicates that alteration of EPO signaling pathway may have an important role in MDS (26).

Alterations in other growth factor pathways have also been reported in MDS patients samples. GM-CSF and G-CSF priming of reactive oxygen species (ROS ) production in neutrophils of patients with MDS is impaired (27). Thrombopoietin signaling has been investigated in the dysmegakaryocytopoiesis seen in MDS, but its role in unclear $(28,29)$.

\section{VAscular Endothelial Growth FACtor}

Vascular endothelial growth factor (VEGF) is a key regulator of angiogenesis. Angiogenesis is increasingly thought to have an important role in MDS and is regulated by multiple signals, including hypoxia inducible factor-1 (HIF-1) and Ras (30). Myelomonocytic precursors of patients with MDS and AML overexpress both VEGF and its high affinity receptor, Flt-1 (31). Inhibition of VEGF reduces leukemia colony formation in clinical samples from MDS patients (32). The importance of VEGF has led to the clinical investigation of VEGF receptor antibodies and VEGF tyrosine kinase inhibitors for the treatment of MDS.

\section{Apoptosis}

Apoptosis is an ordered cellular process that regulates cell population size in a variety of conditions. First described by Wyllie and coll. (33), apoptosis is an energy- dependent process characterized morphologically by cytoplasmic and nuclear condensation, fragmentation of nuclei into "apoptotic bodies", preservation of plasma membrane integrity and phagocytosis of cellular debris by macrophages in the absence of an inflammatory response $(34,35,36)$. This death mechanism is crucial in maintaining a precise number of cells in a given organism. Alterations in apoptosis have been implicated in a variety of medical disorders including myelodysplasia. A variety of stimuli or insults serve as initiators of the apoptotic pathway. These include chemotherapy drugs, ultraviolet and gamma irradiation, chemical exposure, viral infection, steroid hormones, and various cytokines (e.g.,TNF- $\alpha$, Fas ligand, TGF- $\beta$ ) (34).

The process of apoptosis may be conceptually divided into extrinsic and intrinsic pathways. Extrinsic triggers include death ligands (e.g., Fas ligand, TNF- $\alpha$, TNFrelated apoptosis-inducing ligand [TRAIL]) which bind to cell surface receptors and activate downstream signal trasduction pathway. Intrinsic signals that activate the apoptotic pathway result from cellular stress, including exposure to radiation, chemicals or infectious processes. Removal of cellular survival signals (e.g., growth factors) may also trigger intrinsic activations of cell death. Both estrinsic and intrinsic activation of a family of cytosolic aspartate-specific cysteine proteases (caspases). Caspases are the final effector molecules of apoptosis, responsible for the cleavage of both cytosolic and nuclear proteins that result in the stereotypic destruction of the cell.

Evidence for alterations in intramedullary apoptosis in early MDS was first suggested through morphological examination of bone marrow hematopoietic cells. Increased apoptosis of bone marrow progenitors was postulated to account for the clinical observation in early MDS of peripheral blood cytopenias in the presence of a hypercellular bone marrow. It was further postulated that decreased apoptosis may explain the later clinical disease progression and accumulation of immature progenitor cells.

Increased apoptosis in MDS has been shown by morphology, immunohistochemestry, flow cytometry and molecular detection of activated apoptosis-related proteins (37-39). Upregulation of Fas in MDS bone marrow samples has also been reported (40-42). Flow cytometry studies have been utilized to better characterized which specific MDS marrow cells are involved in apoptosis. Using flow cytometric of annexin $\mathrm{V}$ on the surface of apoptotic cells, Parker and coll. (43) demonstrated increased apoptosis in CD34+ cells from early MDS patients compared with late MDS patients. Li et al. (44) showed that apoptosis occurred predominantly (but not exclusively) in non-clonal cells as determined by concurrent FISH in patients with a suitable clonal marker. Rajapaksa and coll. (45) analysed CD34+ and CD34- subfractions of bone marrow from MDS patients and evaluated the sub-diploid (sub-G1) DNA peak after staining with propidium iodide. They observed that the proportion of CD34+cells with sub-G1 DNA (apoptotic) was increased in comparison to normal bone marrow and bone marrow from AML patients. Bcl-2 and c-Myc oncoprotein levels were also evaluated. C-Myc:Bcl-2 oncoprotein ratios were highest in early MDS sample and lower in late MDS and AML samples. The ratio of the pro-apoptotic BAX to anti-apoptotic BCL-2 was increased in early-stage 
MDS but decreased in more advanced disease $(37,46)$. This observation supports the hypothesis that the relative balance between cell-death and cell-survival signals is associated with the increased apoptosis in MDS progenitors. The cause of abnormal apoptosis in MDS is unknown. Both intrinsic cellular defects and extrinsic factors are being investigated in ongoing research. Alterations in the immune-mediated signals, citokine release, and other aspects of bone marrow microenvironment have been implicated.

\section{IMMUNE DYSREGULATION}

There is growing evidence that immune dysregulation plays a role in MDS pathophysiology. The relationship between MDS and autoimmunity stimulated the investigation into the immune system in MDS. The incidence of autoimmune disorders appears to be increased in patients with MDS (47). Autologus cytotoxic T lymphocytes have been observed to exert inhibitory effects on MDS myelopoiesis in vitro. Moreover, the feactures of MDS may overlap with aplastic anemia (AA) and large granular lymphocyte (LGL) disease, two diseases thought to be related to autoreactive $\mathrm{T}$ lymphocytes $(48,49)$. Clinical studies indicated activity of antithymocyte globulin (ATG) and cyclosporine in the treatment of select groups of patients with MDS (50-52). Given the added observation that tumor necrosis factor alpha $(\mathrm{TNF} \alpha) \mathrm{mRNA}$ and protein levels are elevated in both bone marrow and plasma samples of patients with MDS , recent clinical trials have evaluated the efficacy of treatment with immunosuppression and antiTNF therapy (53-57).

Single agent ATG has resulted in complete hematologic responses in up to $10-15 \%$ of patients $(51)$. Predictors of response to immunosuppressive therapy include younger age, presence of a paroxysmal nocturnal hemoglobinuria (PNH) clone, human leukocyte antigen (HLA) DR 15, hypocellularity, and normal karyotype
(58). Responses to ATG have been associated with disappearance of $\mathrm{T}$-cell clones that demonstrate $\mathrm{V}$ beta clonality and which suppress hematopoiesis ex vivo (59). Deeg et al. (53) treated 14 tranfusion-requiring patients with MDS with the combination of ATG and the soluble TNF receptor etanercept. Forty-six percent of patients responded, with five patients achieving periods of red blood cell and platelet independence that exceeded 2 years. These impressive results lend further evidence to the premise that immunomodulation may be effective in select patients with MDS.

Fundamental questions remain unanswered about the precise mechanisms underlying autoimmunity in MDS. The hypothesis that $\mathrm{T}$ lymphocyes attack specific antigens on MDS clonal progenitors remains unproven. Likewise, it is unclear why some patients respond to immunosuppression and others do not. Important future investigations will include confirmation of the efficacy of immunosuppressive and antiTNF therapies in phase III clinical trials and in identifying subsets of patients who will most benefit from these therapies.

\section{Bone Marrow Microenvironment \\ Cytokine Milieu}

The observation of increased bone marrow apoptosis in patients with early MDS stimulated the evaluation of the bone marrow microenvironment as a mediator of MDS pathophysiology. Relative deficiency or overproduction of numerous cytokines, including interleukin $1 \beta(\mathrm{IL}-1 \beta)$, IL-6, IL-8, stem cell factor, erythropoietin, transforming growth factor beta (TGF$\beta)$, GM-CSF and TNF- $\alpha$ have been measured in the bone marrow and serum of patients with MDS with unclear and sometimes conflicting results (60-63). Of all of these cytokines, increased TNF- $\alpha$ has been consistently associated with elevated Fas antigen expression on CD34+ cells. Fas is a membrane protein that

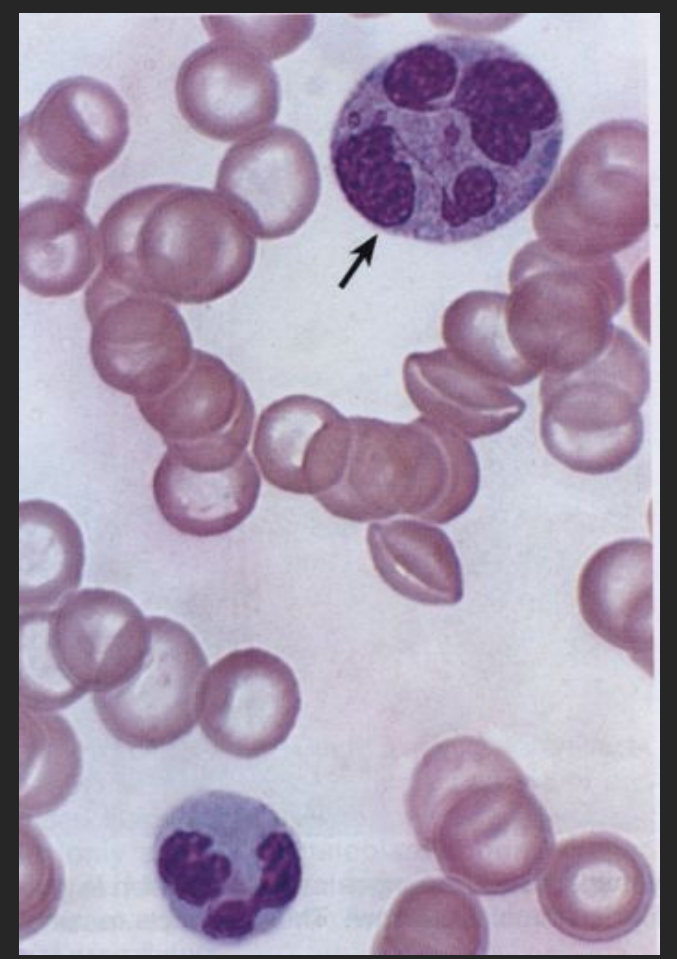

Fig.2 - Neutrophil abnormalities in MDS

Fig.1-Bone marrow picture of a patient with MDS in leukemic trasformation

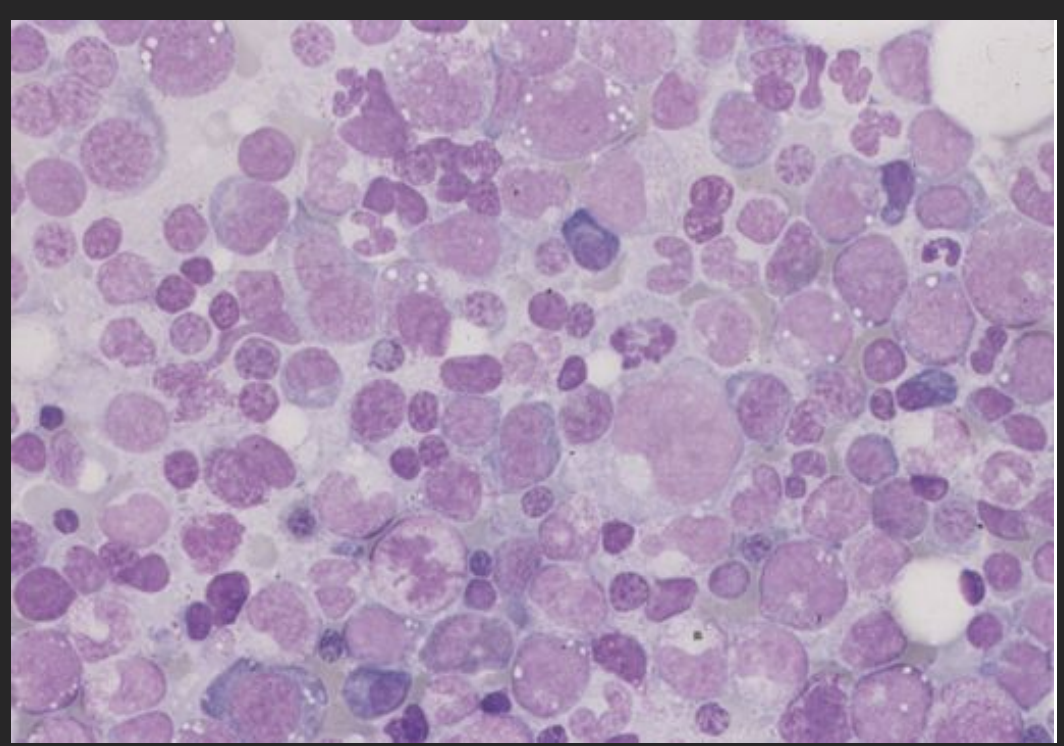


can initiate apoptotic signals in response to crosslinking by the Fas ligand (41). The resultant downstream activation of caspases, which are the critical proteases that result in apoptotic cell death, indicated the importance of elevated TNF- $\alpha$ levels in promoting apoptosis in MDS (64). The source of increased TNF- $\alpha$ is likely marrow macrophages and T-lymhocytes.

\section{NEO-ANGIOGENESIS}

Another aspect of the bone marrow microenvironment that has emerged as an essential factor in pathogenesis of MDS is neo-angiogenesis. Angiogenesis plays a critical role in tumor growth and metastasis (65). Increased microvessel density has been demonstrated in the bone marrow of patients with hematologic malignancies, including MDS (66-68). Neovascularization is mediated by a variety of angiogenic molecules that are released by both tumor cells and normal host cells. Abnormal elevation of several angiogenic cytokines and growth factors in AML samples has been reported. Important molecules include vascular endothelial growth factor (VEGF), basic fibroblast growth factor, (bFGF), angiogenenin, TNF- $\alpha$, and TGF- $\beta$ (69-71). Soluble VEGF receptor has been reported as prognostic factor in both AML and MDS patients (72).

\section{CytOGENETIC AND MOLECULAR ABNORMALITIES}

Cytogenetic abnormalities occur in up to $70 \%$ of patients with primary MDS and to $90 \%$ of patients with therapy-related MDS (5). A well-described difference between primary and secondary MDS is the complexity of abnormal karyotypes. Chromosomal delections are common in MDS as opposed to the balanced translocations that are seen in AML. Over the last decade, intensive investigation has focused on identifying potential tumor suppressor genes in the regions of genetic loss in MDS. Here, we will review some of the insights based on particular chromosomal abnormalities.

\section{Chromosome 5 Deletions}

Approximately $20 \%$ of patients with MDS have abnormalities of chromosome 5 (73). These abnormalities include interstitial delections of the long arm (5q-), monosomy, and unbalanced traslocations. A separate clinical entity of 5q-syndrome has been described in multiple studies in the literature and is now recognized as a separate entity in the WHO classification of MDS (Fig. 3). It is characterized by refractory macrocytic anemia with dyserythropoiesis, a striking female to male ratio of 3:1, normal or high platelet counts, 5q- as the sole cytogenetic abnormality and a low propensity for transformation to AML. In the other cases, abnormalities on chromosome 5 have been seen in famial cases of MDS and in therapy-related MDS $(74,75)$.

The most critical region of deletion is presumed to lie between 5q31 and 5q33. Numerous hematopoietic growth factors are encoded on the long arm of chromosome 5 and loss of these genes is presumed to play a role in MDS pathogenesis. The genes for IL-3, Il-4, IL5 , interferon regulator factor 1 (IRF-1), M-CSF, GM$\mathrm{CSF}$, and the receptor for M-CSF are localized on the long arm of chromosome 5 . These cytokine are critical in the proliferation of granulocytes. However, it is unclear how deletion of any one of these growth factor genes alone would result in a $5 \mathrm{q}$ - syndrome pheno- type.

\section{Chromosome 7 Delections}

Partial or complete deletion of chromosome 7 is a common finding in MDS and AML. It is seen in a variety of settings and is generally associated with poor prognosis. Approximately $10 \%$ of $7 q$-deletions are seen in the setting of "de novo" MDS. The remainder of $7 q-$ delections are seen in cases of MDS arising after environmental or chemotherapeutic exposure and in cases related to familial genetic disorders (e.g. , Fanconi anemia, neurofibromatosis 1 NF1, congenital neutropenia) (75). Analysis from patients with juvenile myelomonocytic leukemia, which often have monosomy 7, has shown that approximately $30 \%$ have NF1 gene mutations (76). NF1 functions as a tumor suppressor gene, encoding a GTPase activation protein acts as a negative regulatior of Ras activity (77). RAS activation occurs in a significant proportion of adult patients with MDS. Gene mutations of RAS or inactivation of the NFl gene are thought to play an important role in the progression of MDS with monosomy 7 (78).

The region at $7 q 22.1$ has been suggested as a critical breakpoint in myeloid malignancies (79). Genes of interest that have been mapped to chromosome $7 \mathrm{q}$ include erythropoietin, plasmin activator inhibitor, T-cell receptor $\beta$, aspargine synthase gene and PIK3CG.

\section{Chromosome 17}

Clinically, deletion of the short arm of chromosome 17 (17p-) is seen primarily in treatment-related MDS and is characterized by dysgranulopoiesis and pseudo-Pelger-Huët anomaly (80). The p53 gene is located at 17p13.1. p53 is a critical tumor suppressor gene that has significant roles in cell cycle control, DNA repair, and apoptosis. Loss of p53 has been documented in a variety of cancers, and it is likely that it plays a role in a subset of MDS cases.

\section{TRISOMY 8}

Trisomy 8 occurs commonly in both acute and chronic leukemias (Fig. 4). A curious observation has been the disappearance of trisomy 8 clones in the course of the disease. This phenomenon is independent of the percentage of blasts in the bone marrow or the clinical status of disease $(81,82)$. As such, it is unclear how significant trisomy 8 is in the pathogenesis of MDS, and therefore, it has not attracted as much attention as some of other cytogenetic abnormalities.

\section{Other Chomosomal Deletions}

Loss of portions of chromosomes 3, 11, 12, 13, and the Y chromosome have been described with varying frequency in the literature. Likewise, trisomies involving chromosomes 6,13 and 21 have been documented. Although critical regions on each chromosome have been identified and a number of candidate tumor suppressor genes have been identified, the molecular pathogenesis remains elusive.

\section{REFERENCES}

1. Steensma DP, Tefferi A: The myelodysplastic syndrome(s): a perspective and review highlighting current controversies. Leuk Res 27:95-120, 2003. 


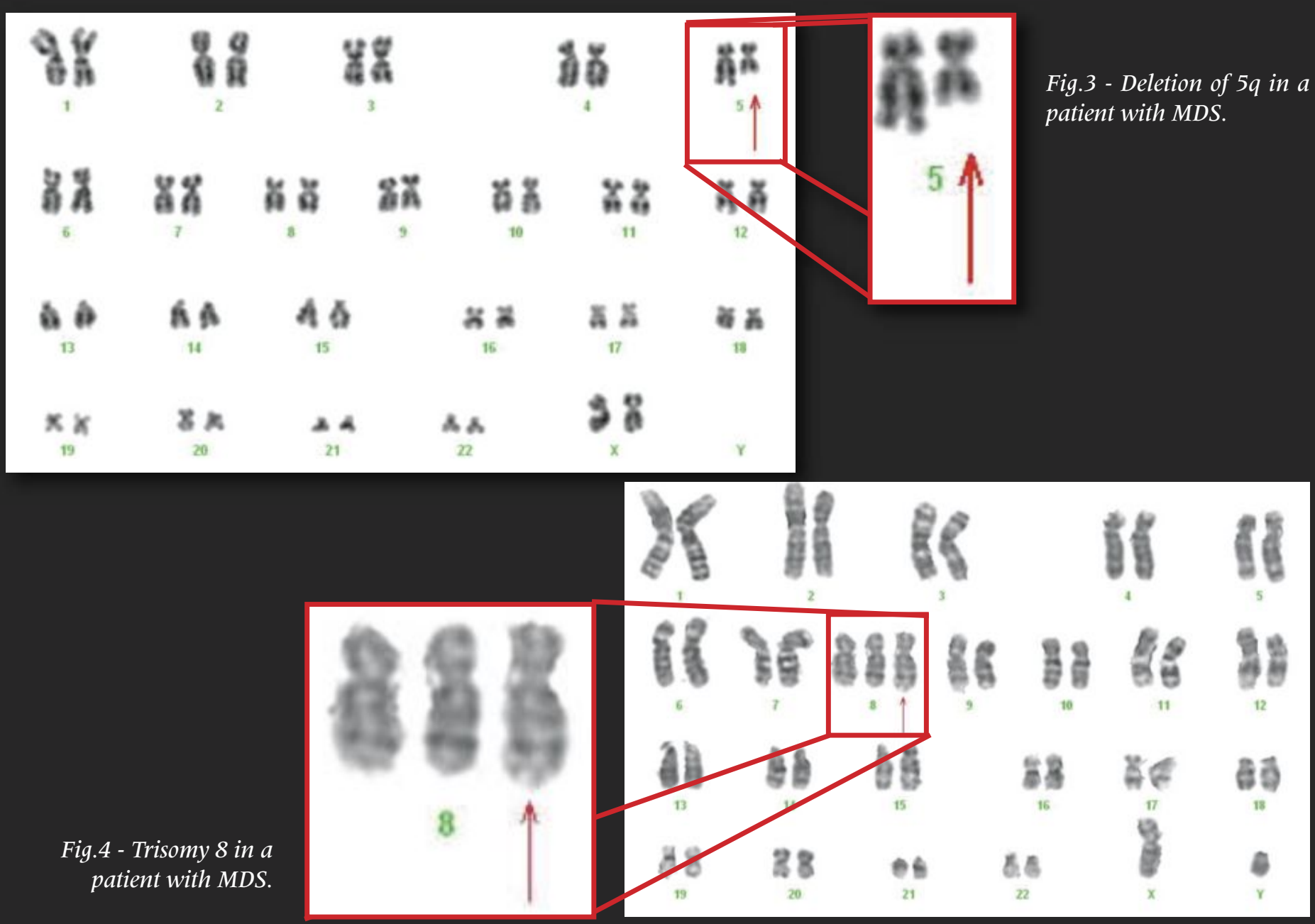

2. Bennett JM, Catovsky D, Daniel MT et al.: Proposals for the classification of the myelodysplatic syndromes. Br J Haematol 51: 189-199, 1982

3. Harris NL, Jaffe ES, Diebold J et al.: World Health Organization classification of neoplastic diseases of hematopoietic and lymphoid tissue: report of the clinical advisory committee meeting, Airlie House, Virginia, November 1997. J Clin Oncol 17:3835-3849, 1999

4. Buckle VJ, Wainscoat JS: Clonality of cell populations in refractory anemia using combined approach of gene loss and $X$-linked restriction fragment length polymorphism-methylation analyses. $\mathrm{Br} \mathrm{J}$ Haematol 79:550-555, 1991

5. Delforge M. Understanding the pathogenesis of myelodysplastic syndromes (Review). Hematol J 4:303-309, 2003

6. Nilsson L, Astrad-Grundstrom I, Anderson K et al.: Involvement and functional impairment of the CD34+CD38-Thy-1+ hematopoietic stem cell pool in myelodysplastic syndromes with trisomy 8. Blood 100:259267 (2002)

7. Brillet JM, Lejeune R, Fenaux P: Occupational and environmental risk factors of the myelodysplastic syndromes in North of France. Br J Haematol 112:927-935, 2001

8. Garfinkel L, and Boffetta P: Association between smoking and leukemia in two American Cancer Society prospective studies. Cancer 65:2356-2360, 1990

9. Agostini P, De Angeli C, Del Senno L et al.: Expoure to myelotoxic agents and myelodysplasia: case-control study and correlation with clinicobiological findings. Br J Haematol 130:189-197, 1998

10. West RR, Stafford DA, Farrow A et al.: Occupational and environmental exposures and myelodysplasia: a case-control study. Leuk Res 19:127-139, 1995

11. Engelhardt M, Wasch R, Guo Y: Telomeres and telomerase in normal and leukemic hematopoietic cells. Leuk Res 28:1001-1004, 2004

12. Ohyashiki JH, Ohyashiki K, Fujimura T et al.: Telomere shortening associated with disease evolution patterns in myelodysplastic syndromes. Cancer Res 54: 3557-3560, 1994

13. Henikoff S, and Matzke MA: Exploring and explaining epigenetic effects. Trends Genet 13:293-295, 1997

14. Attwood JY, Yung RL, Richardson BC: DNA methylation and the regulation of gene trascription (Review). Cell Mol Life Sci 59:241-257, 2002

15. Nakayama M, Wada M, Harada $T$ et al.: Hypomethylation status of $C p G$ site at the promoter region and overexpression of human MDRI gene in acute myeloid leukemias. Blood 92:4296-4307, 1998

16. Tsukamoto N, Morita K, Karasawa $M$ et al.: Methylation status of c-myc oncogene in leukemic cells: hypomethylation in acute leukemia derived from myelodysplastic sindrome. Exp Hematol 20:1061-1064, 1992

17. Quesnel B, Guillerm $G$, Vereecque $R$ et al.: Methylation of the p15(INK4b) gene in myelodysplastic syndromes is frequent and acquired during disease progression. Blood 91:2985-2990, 1998

18. Uchida T, Kinoshita T, Nagai $H$ et al.: Hypermethylation of p15INK4B gene in myelodysplastic syndromes. Blood 90:1403-1409, 1997

19. Esteller m, Herman JG: Cancer as an epigenetic disease: DNA methylation and chromatin alterations in human tumours (Review). J Pathol 196:1-7, 2002

20. Herman JG, Baylin SB: Gene silencing in cancer in association with promoter hypermethylation (Review) N Engl J Med 349:2042-2054, 2003

21. Backx B, Broeders L, Touw I: Blast colony-forming cells in myelodysplastic syndrome: decreased potential to generate erythroid precursors. Leukemia 7:75-79, 1993

22. Mayani $H$, Baines $P$ Bowen DT et al: In vitro growth of myeloid and erythroid progenitor cells from myelodysplastic patients in response to recombinat human granulocyte-macrophage colony-stimulating factor. Leukemia 3:29-32, 1989

23. Tanner JW, Chen W, Young RL et al.: The conserved box 1 motif of cytokine receptors is receptors is required for association with JAK kinase. J Biol Chem 270:6523-6530, 1995

24. Witthuhn BA, Quelle FW, Silvennoinen et al.: JAK2 associates with the erythropoietin receptor and is tyrosine phosphorylated following 
stimulation with erythropoietin. Cell 74:227-236, 1993

25. Hoogerbrugge H, Touw IP, Lowenberg B: Erythropoietin-induced activation of STAT5 is impaired in the myelodysplastic syndrome. Blood 89:1690-1700, 1997

26. Backx B, Broeders L, Hoefsloot LH: Erythropoiesis in myelodysplastic syndrome : expression of receptors for erythropoietin and kit ligand. Leukemia 10:466-472, 1996

27. Fuhler GM, Drayer AL, Vellenga E: Decreasead phosphorylation of protein kinase $B$ and extracellular signal-regulated kinase in neutrophils from patients with myelodysplasia. Blood 101:1172-1180, 2003

28. Hofmann Wk, Kalina U, Koschmieder S et al.: Defective megakaryocytic development in myelodysplastic syndromes (Review). Leuk Lymphoma 38:13-19, 2000

29. Kalina U, Hofmann WK, Koschmieder S et al.: Alteration of c-mplmediated signal transduction in CD34(+) cells from patients with myelodysplastic syndromes. Exp Hematol 28:1158-1163, 2000

30. Estey EH: Modulation of angiogenesis in patients with myelodysplastic syndrome. Baillier's Best Practice in Clinical Haematology 17:623-639, 2004

31. Bellamy WT, Richter L, Sirjani D et al.: Vascular endothelial cell growth factor is an autocrine promoter of abnormal localized immature myeloid precursors and leukemia progenitor formation in myelodysplastic syndromes. Blood 97:1427-1434, 2001

32. Broxmeyer HE, Cooper $S, \mathrm{Li} \mathrm{ZH}$ et al.: Myeloid progenitor cell regulatory effects of vascular endothelial cell growth factor. Int J Hematol 62:203-215,1995

33. Wyllie AH, Kerr JF, Currie AR: Cell death: the significance of apoptosis. Int Rev Cytol 68:251-306, 1980

34. Greenberg PL: Apoptosis and its role in the myelodysplastic syndromes: implications for disease natural history and treatment (Review) Leuk Res 22:1123-1136, 1998

35. Walker NI, Harmon BV, Gobe GC et al.: Patterns of cell death (review). Methods $\theta$ Achievements in Experimental Pathology 13:18-54, 1988;

36. Wyllie AH :The biology of cell death in tumours. Anticancer Res 5:131-136,1985

37. Parker JE, Mufti Gj: The role of apoptosis in the pathogenesis of the myelodysplastic syndromes (Review). Int J Hematol 73:416-428, 2001

38. Raza A, Gezer S, Mundle S et al.: Apoptosis in bone marrow biopsy sample involving stromal and hematopoietic cells in 50 patients with myelodysplastic syndromes. Blood 86: 268-276, 1995a

39. Raza A, , Mundle S, Iftikhar A et al.: Simultaneous assessment of cell kinetics and programmed cell death in bone marrow biopsies of myelodysplastics reveals extensive apoptosis as the probable basis for ineffective hematopoiesis. Am J Hematol 48:143-154, 1995b

40. Bouscary D, De Vos J, Guesnu M et al. : Fas/Apo-1 (CD95) expression and apoptosis in patients with myelodysplastic syndromes. Leukemia 11:839-845,1997

41. Gersuk GM, Beckman C Loken MR et al : A role of tumor necrosis factor- $\bigotimes$, Fas and Fas-Lingard in marrow failure associated with myelodysplastic syndrome. Br J Haematol 103:176-188, 1998

42. Kitagawa M, Yamaguchi S, Takahashi M et al.: Localization of Fas and Fas lingand in bone marrow cells demonstrating myelodysplasia. Leukemia 12:486-492, 1998

43. Parker Je, Fishlock KL, Mijovic A et al.: "Low risk" myelodysplastic syndrome is associated with apoptosis and an increased ratio of proversus anti-apoptotic bcl-2-related proteins. Br J Haematol 103:10751082,1998

44. Li X, Bryant E, Deeg HJ: Simultaneous demonstration of clonal chromosome abnormalities and apoptosis in individual marrow cells in myelodysplastic syndrome. Int J Hematol 80:140-145, 2004

45. Rajapaksa R, Ginzton N, Rott LS et al. : Altered oncoprotein expression and apoptosis in myelodysplastic syndrome marrow cells. Blood 88:4275-4287, 1996

46. Parker JE, Mufti GJ, Rasool F et al. : The role of apoptosis, proliferation, and $\mathrm{Bcl}-2$-related proteins in the myelodyslastic syndromes and acue myeloid leukemia secondary to MDS. Blood 96:3932-3938, 2000 47. Saif MW, Hopkins JL, Gore SD: Autoimmune phenomena in patients with myelodisplastic syndromes and chronic myelomonocytic leukaemia (Review). Leuk Lymphoma 43:2083-2092, 2002

48. Barrett J, Sauthararajah Y, Molldrem J: Myelodysplastic syndrome and aplastic anemia: distinct entities or diseases linked by a common pathophysiology? (Review) Semin Hematol 37:15-29, 2000

49. Kanchan K, Loughran TP: Antigen-driven clonal T cell Expansion in disorders of hematopoiesis. Leuk Res 27:291-292, 2003

50. Jonasova A, Neuwitova $R$, Cermak J, et al. Cyclosporin. A therapy in hypoplastic MDS patients and certain refractory anaemias without hypoplastic bone marrow. Br J Haematol 100:304-309,1998

51. Killick SB, Mufti G, Cavenagh JD et al.: A pilot study of antithymocyte globulin (ATG) in the treatment of patients with "low-risk" myelodysplasia. Br J Haematol 120:679-684, 2003

52. Molldrem JJ, Leifer E, Bahceci E, et al.: Antithymocyte globulin for treatment of bone marrow failure associated with myelodysplastic syndrome [ summary for patients in Ann Intern Med 2002 137:1-27]. Ann Intern Med 137:156-163, 2002

53. Deeg HJ, Jiang PYZ, Holmberg LA: Hematologic responses of patients with MDS to antithymocyte globulin plus etanercept correlate with improved flow scores of marrow cell. Leuk Res 28:1177-1180, 2004 54. Kitagawa $T$, Kamiyama $R$, Hirokawa $K$ et al.: Overexpression of tumor necrosis factor (TNF)-alpha and interferon (IFN)-gamma by bone marrow cells from patients with myelodysplastic syndromes. Leukemia 11:2049-2054, 1997

55. Maciejewski J, Selleri C, Anderson $S$ et al.: Fas antigen expression on CD34+ human marrow cellsis induced by interferon gamma and tumor necrosis factor alpha and potentiates cytokine-mediated hematopoietic suppression in vitro. Blood 85:3183-3190, 1995

56. Molnar L, Berki T, Hussain A et al. : Detection of TNFalpha expression in the bone marrow and determination of TNFalpha production of peripheral blood mononuclear cells in myelodysplastic syndrome. Path. Oncol. Res 6:18-23, 2000

57. Rosenfeld C, Bedell C: Pilot study of recombinant human soluble tumor necrosis factor receptor (TNFR:FC) in patients with low risk myelodysplastic syndrome. Leuk Res 26.721-724, 2002

58. Saunthararajah $Y$, Nakamura $R$, Nam JM et al. : HLA-DR15 (DR2) is overrepresented in myelodysplastic syndrome and aplastic anemia and predicts a response to immunosuppression in myelodysplastic syndrome. Blood 100:1570-1574, 2002

59. Kochenderfer JN, Kobayashi S, Wieder ED et al.:Loss of T-lymphocyte clonal dominance in patients with myelodysplastic syndrome responsive to immunosuppression. Blood 100:3639-3645, 2002

60. Bowen D, Yancik S, Bennett L et al. : Serum stem cell factor concentration in patients with myelodysplastic syndromes. Br J Haematol 85:63-66, 1993

61. Fontenay-Roupie M, Bouscary D, Guesnu M et al. : Ineffective erythropoiesis in myelodysplastic syndromes: correlation with Fas expres sion but not with lack of erythropoietin receptor signal trasduction. $\mathrm{Br}$ J Haematol 106:464-473, 1999

62. Maurer AB, Ganser A, Buhl R et al.: Restoration of impaired cy tokine secretion from monocytes of patients with myelodysplastic syndromes after in vivo treatment with GM-CSF or IL-3. Leukemia 7:1728-1733,1993

63. Verhoef GE, De Schouwer P, Ceuppens JL et al.: Measurement of serum cytokine levels in patients with myelodysplastic syndromes. Leukemia 6:1268-1272,1992

64. Mundle Sd, Reza S, Ali A et al.: Correlation of tumor necrosis factor alpha (TNF alpha) with high Caspase 3-like activity in myelodysplastic syndromes. Cancer Lett 140:201-207,1999

65. Folkman J: Angiogenesis in cancer, vascular, rheumatoid and other disease (Review). Nat Med 1:27-31,1995

66. Alexandrakis MG, Passam FJ, Ganotakis E et al.: Bone marrow microvascular density and angiogenic growth factors in multiple myeloma. Clin Chem Lab Med 42:1122-1126,2004

67. Padro T, Ruiz S, Bieker $R$ et al.: Increased angiogenesis in the bone marrow of patients with acute myeloid leukemia. Blood 95:2637-2644, 2000

68. Pruneri G, Bertolini F, Soligo D et al.: Angiogenesis in myelodysplastic syndromes. $\mathrm{Br} J$ Cancer 81:1398-1401, 1999

69. Albitar M: Angiogenesis in acute myeloid leukemia and myelodys plastic syndrome (Review). Acta Haematol 106.170-176, 2001

70. Alexandrakis MG, Passam FH, Pappa CA et al.: Relation between bone marrow angiogenesis and serum levels of angiogenin in patients with myelodysplastic syndromes. Leuk Res 29:41-46, 2005

71. Faderl S, Kantarjian HM: Novel therapies for myelodysplastic syndromes (Review). Cancer 101:226-241, 2004

72. Hu $Q$, Dey AL, Yang $Y$, et al. : Soluble vascular endothelial growth factor receptor 1 , and not receptor 2 , is an independent prognostic factor in acute myeloid leukemia and myelodysplastic syndromes. Cancer 100:1884-1891,2004

73. Third MIC Cooperative Study Group: Recommendations for a morphologic, immunologic, and cytogenetic (MIC) working classification of the primary and therapy-related myelodysplastic disorders. Report of the workshop held in Scottsdale, Arizona, USA. On Febrary 23-25, 1987. Third MIC Cooperative Study Group. Cancer Genet Cytogenet 32:1-10, 1998

74. Grimwade DJ, Stephenson J, De Silva C et al. : Familial MDS with 5q-abnormality. Br J Haematol 84:536-538,1993

75. Mhawech P, Saleem: A Myelodysplastic syndrome: review of the cy togenetic and molecular data (Review). Critical Reviews in OncologyHematology 40:229-238, 2001

76. Shannon KM, O'Connel $P$, Martin $G A$ et al.: Loss of normal NF1 allele from the bone marrow of children with type 1 neurofibromatosis and malignant myeloid disorders. N Engl J Med 330:597-601, 1994

77. Martin GA, Viskochil D, Bollag G et al. : The GAP-related domain of the neurofibromatosis type 1 gene product interacts with ras $p 21$. Cell 63:843-849,1990

78. Stephenson J, Lizhen H, Mufti GJ: Possible co-existence of RAS activation an monosomy 7 in the leukemic trasformation of myelodysplastic syndromes. Leuk Res 19:741-748, 1995

79. Johnson EJ, Scherer SW, Osborne L et al. : Molecular definition of a narrow interval at 7q22.1 associated with myelodysplasia. Blood $87: 3579-3586,1996$

80. Lai JL, Preudhomme C, Zandecki M et al. : Myelodysplastic syndromes and acute myeloid leukemia with $17 p$ deletion. An entity char acterized by specific dysgranulopoiesis and a high incidence of P53 mutations. Leukemia 9:370-381, 1995

81. Iwabuchi A, Ohyashiki JH, Ohyashiki JH et al.: Trisomy of chromosome 8 in myelodysplastic syndrome. Significance of the fluctuating trisomy 8 population. Cancer Genet Cytogenet 62:70-74, 1992

82. Matsuda A, Yagasaki F, Jinnai I et al. : Trisomy 8 may not be related to pathogenesis of myelodysplastic syndromes: disappearance of trisomy 8 in a patients with refractory anaemia without haematological improvement. Eur J Haematol 60:260-261, 1998 